\title{
Persistent Buttock Claudication after Endovascular Abdominal Aortic Aneurysm Repair
} A Surgical Solution

\author{
Alessandro Robaldo, MD*, Stefano Pagliari, MD, Filippo Piaggio, MD, Patrizio Colotto, MD \\ Vascular and Endovascular Surgery Unit, Imperia Hospital, Imperia, Italy
}

\begin{abstract}
We describe the successful surgical treatment of a 71-year-old man affected by right buttock claudication after a right internal iliac artery (IIA) coil embolization as an adjunct to endovascular iliac artery aneurysm repair. Computed tomography angiography revealed extensive aortoiliac calcifications and thrombus in the vessel walls. Despite patency of the contralateral IIA and preservation of right distal collateral flow through ipsilateral hypogastric branches, the symptom was persistent and disabling. The high-risk patient underwent an "open" repair of the infrarenal abdominal aneurysm with removal of the entire stent-graft and concomitant revascularization of the right IIA. Post-operative recovery was uneventful, and the patient remained asymptomatic during a 30-month follow-up. This case underscores the importance of considering all potential solutions, including open surgery, to preserve pelvic inflow after aortoiliac stent grafting, particularly for high-risk patients with vulnerable plaque and higher risk of thrombus embolization.

Copyright $\odot 2017$ Science International Corp.
\end{abstract}

\section{Key Words:}

Buttock claudication - Coil embolization - Internal iliac artery $•$ Endovascular abdominal aortic aneurysm repair

\section{Introduction}

Buttock claudication is the most frequent ischemic complication after hypogastric artery occlusion prior to endovascular abdominal aortic aneurysm repair.

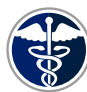

Fax +1 2037853552

E-Mail: aorta@scienceinternational.org

http://aorta.scienceinternational.org

\author{
(c) 2017 AORTA \\ Published by Science International Corp. \\ ISSN 2325-4637 \\ Accessible online at: \\ http://aorta.scienceinternational.org
}

The English literature contains conflicting reports of the incidence and severity of symptoms. While many authors find internal iliac artery (IIA) coil embolization a relatively trouble-free procedure, there are reports of devastating sequelae and mortality associated with the technique [1, 2]. A variety of new endovascular devices and surgical procedures have been proposed for situations in which the hypogastric artery must be covered by the iliac limb endograft $[3,4]$. The aim of this report is to present a successful surgical technique of hypogastric artery bypass to treat persistent buttock claudication occurring after endovascular common IIA repair with unilateral IIA interruption.

\section{Case Presentation}

A-71-year-old man with a history of heart failure, hypertension, peripheral arterial disease, and hypercholesterolemia presented to our department for persistent and disabling right buttock claudication. The symptom occurred subsequent to ipsilateral IIA coil embolization performed $24 \mathrm{~h}$ prior to endograft limb extension to the external iliac artery (EIA) as an adjunct to endovascular repair of a common iliac artery aneurysm. No antecedent back trauma or history of prolapsed lumbar discs was reported.

Abdominal duplex scan showed complete occlusion of the right IIA with stent-graft exclusion of the right common iliac artery aneurysm. Patency of the contralateral IIA was detected. No

\footnotetext{
* Corresponding Author:

Alessandro Robaldo, MD

Vascular and Endovascular Surgery Unit Imperia Hospital

Via Sant'Agata, 57 18100, Imperia, Italy

Tel.: +39 0183 537214; Fax: +39 0183 537317; E-Mail: robaldo.alessandro@gmail.com
} 

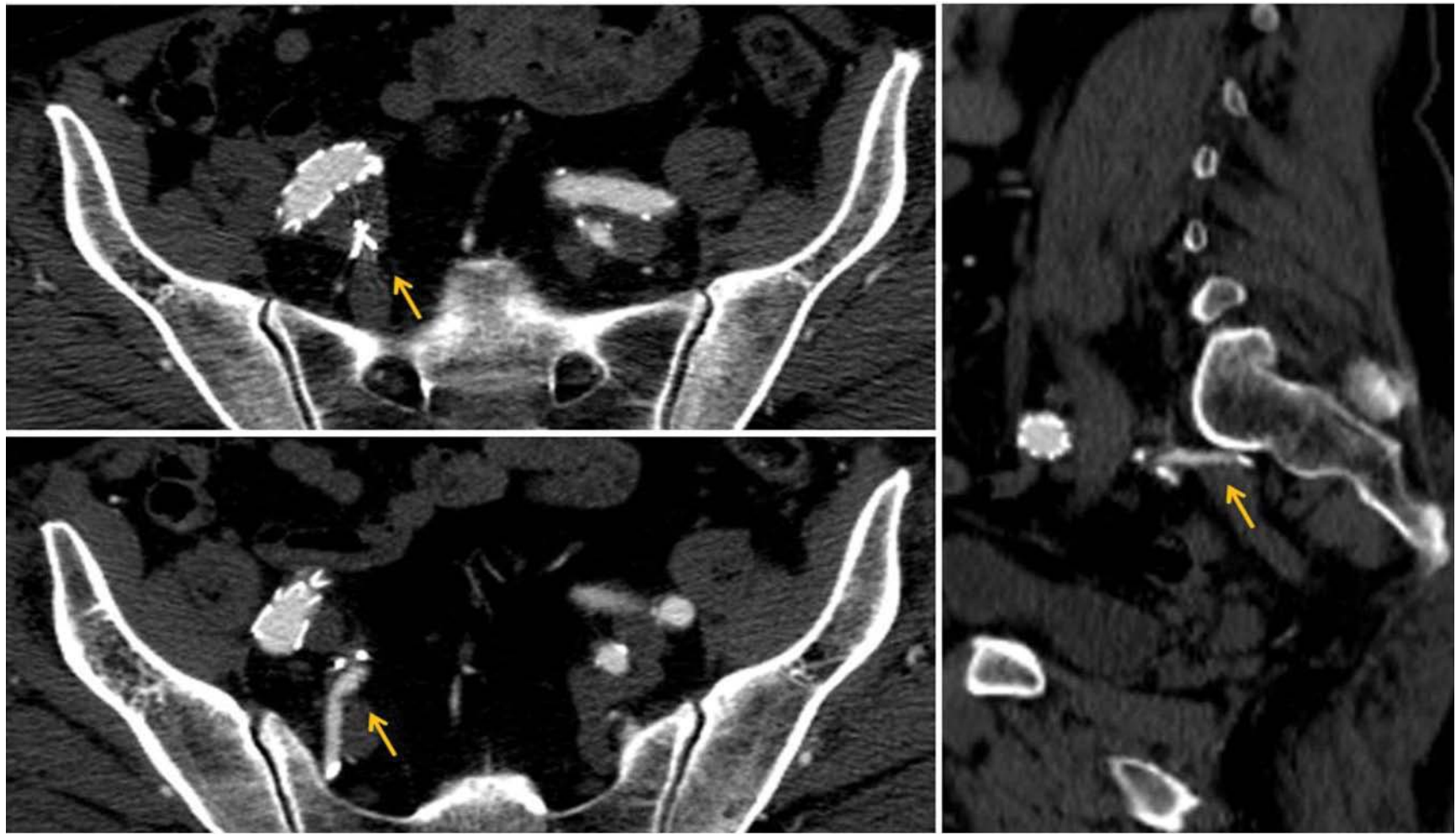

Figure 1. Preoperative computed tomography angiography. Axial and sagittal images demonstrating proximal coil placement into the iliac bifurcation and patency of the distal portion of the right internal iliac artery (arrows).

other aneurysm sites or aortoiliac stenotic lesions were recognized. Continuous wave Doppler revealed a signal at the right buttock level with a lower intensity than that of the contralateral side. Contrast-enhanced multidetector computed tomography angiography confirmed the diagnosis and showed patency of the distal portion of the right IIA and its branches, with preserved distal collateral flow (Figure 1). Additional findings included severe aortic and iliac calcifications and mural thrombus. Preoperative cardiac testing did not detect atrial fibrillation and the patient was considered to have grade III preoperative risk according to American Society of Anesthesiologists classification.

Under general anesthesia, through median transperitoneal access and infrarenal clamping, the stentgraft and IIA coil were removed. After retrograde right hypogastric blood flow was assessed, revascularization was performed in two steps. First, a bifurcated Dacron graft was anastomosed in an end-to-end fashion from the infrarenal aorta to the left common iliac artery and the distal part of the right IIA, respectively. Second, a separate graft, proximally anastomosed end-to-side with the corresponding anterior aspect of the main body of the aorto iliac graft, was jumped to the right EIA (Figure 2).

Post-operative recovery was uneventful. The patient was discharged in good general condition with complete pain relief on life-long mono-antiplatelet treatment (acetylsalicylic acid $300 \mathrm{mg}$ ). At 30-month follow-up, there was no buttock claudication, pelvic ischemia, or complaint of paresthesias, pain, discomfort, or walking limitation. Computed tomography angiography showed patency of both grafts with no anastomotic stenosis/pseudoaneurysms. The right IIA at the level of its first branch was well perfused (Figure 3).

\section{Discussion}

Pooled data from different studies reveal that the incidence of buttock claudication after coil embolization of one or both IIAs ranges from $11 \%$ to $50 \%$, with 

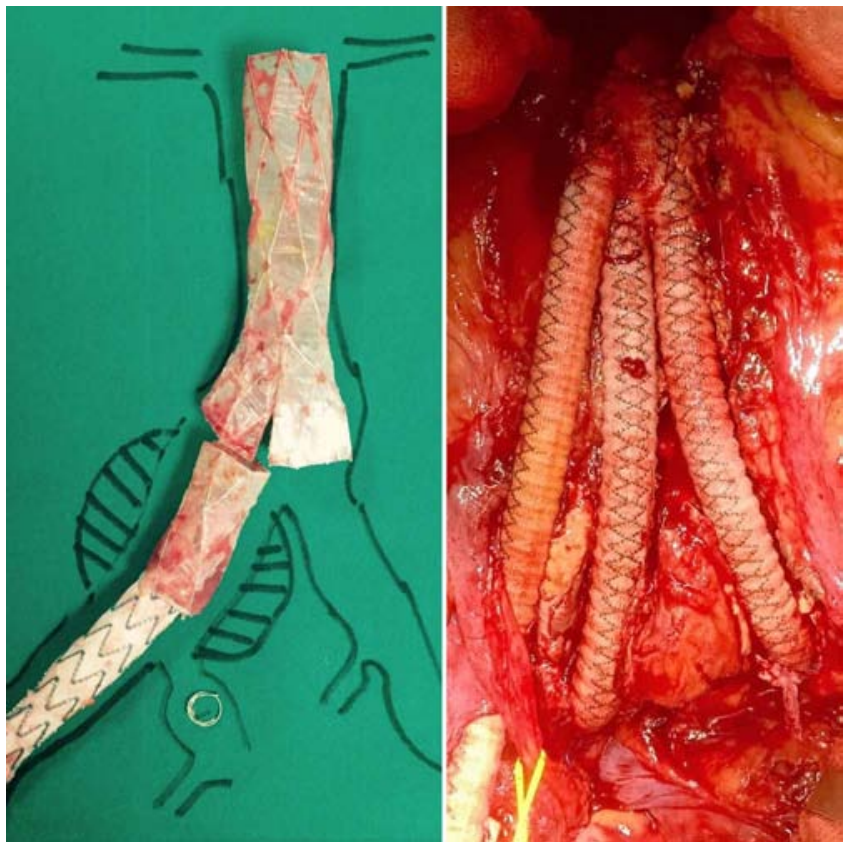

Figure 2. The left image shows the removed stent-graft and embolization coil placed on a schematic design of the previous implant. The right images show intraoperative surgical direct aortoiliac revascularization of the right internal iliac artery and both common iliac arteries via a transperitoneal approach.

differences in type and severity [1]. Persistent buttock claudication represents approximately $13 \%$ of all of IIA coil embolization procedures, with no statistically significant difference between unilateral and bilateral IIA interruption [2]. Although some studies report that IIA embolization is a well-tolerated procedure with a small chance of severe morbidity, recent literature appears to support the notion that every effort must be made to preserve one or both hypogastric arteries during endovascular aneurysm repair $[3,4]$.

This report demonstrates the potentially problematic nature of the management of the disabling sequelae of this complication. Despite the fact that the IIA embolization was performed in a staged fashion and the coil placement was proximal to the internal iliac bifurcation with evidence of distal patent collateral vessels and consequent time to develop a collateral pelvic circulation with blood flow from the contralateral IIA and ipsilateral EIA, the patient experienced constant and painful buttock claudication. Likely, periprocedural distal embolization or a hypotension episode may have contributed to the onset of the early buttock claudication [5].

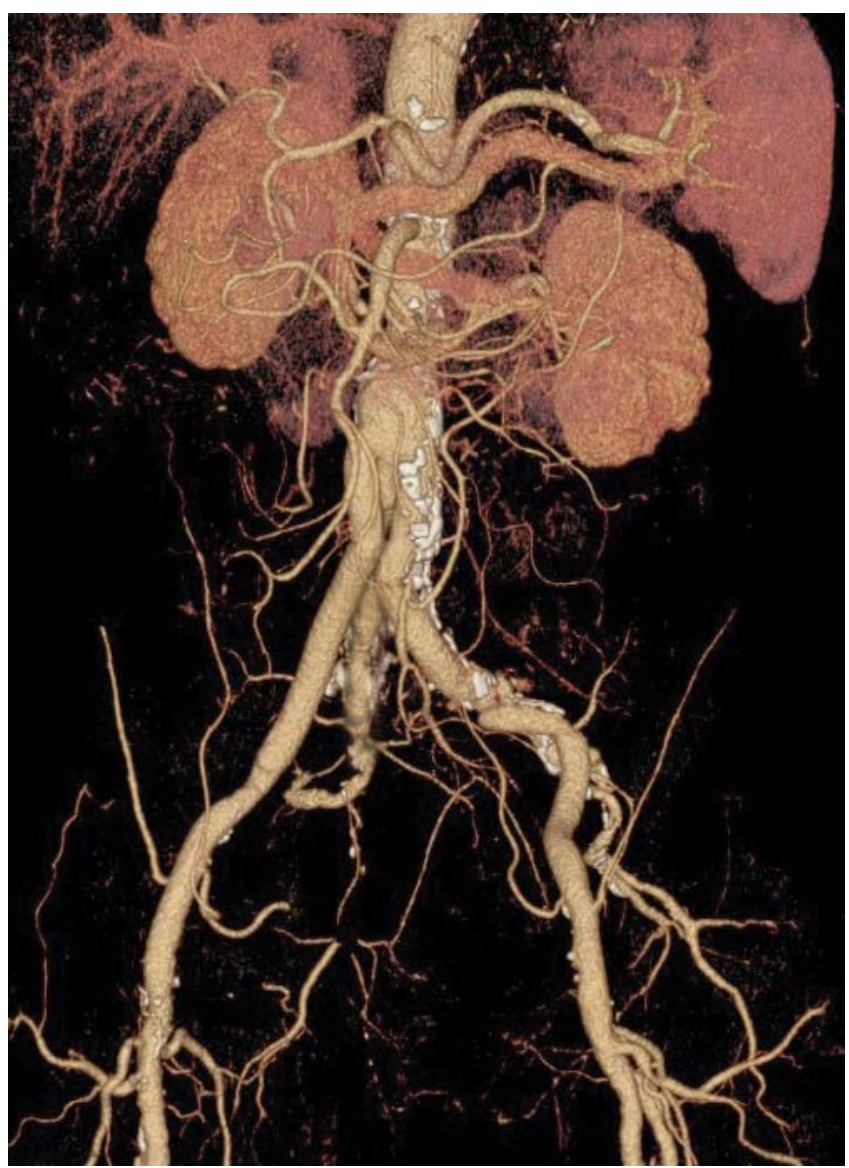

Figure 3. Follow-up computed tomography angiography at 6 months showed a good result of the procedures with patency of all branches of the graft without further complications.

Different surgical solutions have been designed to restore hypogastric perfusion. Many surgeons prefer a retrograde IIA revascularization with relocation of the IIA to the external iliac or femoral arteries via a retroperitoneal approach to avoid extensive pelvic dissection, reduce trauma to the abdominal wall musculature, and achieve less postoperative patient discomfort [6]. However, in our case, we believe the best choice to restore buttock circulation was to perform direct revascularization. In fact, retrograde perfusion may be inadequate for many reasons. First, the short available segment of the EIA due to extension of the endograft limb and the deep location of the patent IIA vessel branches can make the surgical area uncomfortable and the entire procedure technically complicated, with a risk of injury to large veins. Second, perfusion may 
be not achieved in case of severe atherosclerotic external deterioration of the iliac-femoral junction. Finally, prolonged clamping of the EIA associated with difficult control of intraoperative activated clotting time during performance of the anastomosis can cause potential peri-postoperative limb thrombosis.

Based on these considerations, whenever possible and reasonable according to American Society of Anesthesiologists score, our choice has been to perform direct bypass from the aorta to the IIA with an end-to-end anastomosis between a branch of a bifurcated Dacron graft and the residual distal part of the hypogastric artery via a transperitoneal approach. We believe that this offers better exposure of distal IIA branches. From a technical point of view, to perform an "octopus" trifurcated graft, as described above, with a separate jump to the EIA from the main body graft shows some advantages. First, unless a poor runoff or quality of the artery wall were intraoperatively detected, this configuration can result in an optimal perfusion pressure of each target vessel without any risk of steal syndromes. Moreover, in case of occlusion of the IIA branch, there is reduced risk of thrombotic involvement of the anastomosed branch to the EIA. Finally, the anastomosis achieved on the anterior aspect of the main body is technically easier and provides better perioperative control of bleeding when compared with an end-to-side proximal anastomosis of a separate IIA graft to the corresponding posterior side of an aorto-iliac prosthesis branch. Although treatment should be individualized, we recommend this surgical approach in cases of persistent buttock claudication to restore hypogastric artery circulation after IIA coil embolization.

\section{Conflict of Interest}

The authors have no conflict of interest relevant to this publication.

\section{Comment on this Article or Ask a Question}

\section{References}

1. Hye Ryeon C, Ki Hyuk P, Jae Hoon L. Risk factor analysis for buttock claudication after internal iliac artery embolization with endovascular aortic aneurysm repair. Vasc Specialist Int. 2016;32:44-50. DOI: 10.5758/ vsi.2016.32.2.44

2. Mehta M, Veith FJ, Ohki T, Cynamon J, Goldstein K, Suggs WD. Unilateral and bilateral hypogastric artery interruption during aortoiliac aneurysm repair in 154 patients: a relatively innocuous procedure. J Vasc Surg. 2001;33:S27-S32. DOI: 10.1067/ mva.2001.111678

3. Faries PL, Morrissey N, Burks JA, Gravereaux E, Kerstein MD, Teodorescu VJ, et al. Internal iliac artery revascularization as an adjunct to endovascular repair of aortoiliac aneurysms. J Vasc Surg. 2001;34:892899. DOI: $10.1067 / \mathrm{mva} .2001 .118085$

4. Karthikesalingam A, Hinchliffe RJ, Holt PJ, Boyle JR, Loftus IM, Thompson MM. Endovascular aneurysm repair with preservation of the internal iliac artery using the iliac branch graft device. Eur J Vasc Endovasc Surg. 2010;39:285-294. DOI: 10.1016/j. ejvs.2009.11.018

5. Maldonado TS, Rockman CB, Riles E, Douglas $D$, Adelman MA, Jacobowits GR, et al. Ischemic complications after endovascular abdominal aortic aneurysm repair. J Vasc Surg. 2004;40:703-709. DOI: 10.1016/j. jvs.2004.07.032
6. Milite D, Campanile F, Tosato F, Pilon F, Zaramella M. Hypogastric artery bypass in open repair of abdominal aortoiliac aneurysm: a safe procedure. Interact Cardiovasc Thorac Surg. 2010;10:749-752. DOI: 10.1510/icvts.2009.216846

Cite this article as: Robaldo A, Pagliari S, Piaggio F. Persistent Buttock Claudication after Endovascular Abdominal Aortic Aneurysm Repair: A Surgical Solution. AORTA (Stamford). 2017;5(6):173-176. DOI: https://doi.org/10.12945/j. aorta.2017.17.036 